\title{
Amadeus
}

International Multidisciplinary Journal ISSN 2525-8281

V.2, N. 4. Jul./2018 - 10.14295/aimj.v2i4.38. ISSN 2525-8281

\section{THE INNOVATIONS, ADVANCES AND OUTLOOKS OF LIQUID BIOPSY IN ONCOLOGY: A LITERATURE REVIEW}

Antonio Marlos Duarte de

Melo ${ }^{1}$;

Messias Silvano da Silva

Filho $^{2}$;

Bárbara Torquato Alves ${ }^{2}$;

Kevellyn Cruz Aguilera ${ }^{2}$;

Ana Maria Correia Alencar ${ }^{2}$; Ana Maria Lima Carneiro de

Andrade;

Daniel Gonçalves Leite ${ }^{2}$; Ricardo Souto Quidute

\begin{abstract}
Studies on the biology of cancer are multiplying and have been giving significant repercussions on the care of cancer patients, and there is a growing need to evaluate the biology of the tumor. Conventional tissue biopsies currently represent the gold standard in the diagnosis of cancer, but they are not suitable for serial analysis because of the need for invasive procedures, besides being able to present a high risk of life and also impossibility of reaching surgical in some tumors. To solve this obstacle, the use of the Liquid Biopsy, which analyzes the presence of biomarkers released by cancer cells, such as circulating tumor cells (CTCs), tumor cell DNA (ctDNA) and exosomes is being discussed. These techniques are noninvasive or minimally invasive and collect their samples from peripheral blood, plasma and serum, urine, saliva and cerebrospinal fluid (CSF). As they are already being used in the treatment of several histopathological types of cancer, these new techniques generally represent a revolution in the understanding of early diagnosis, choice of personalized treatment, followup of the treatment response in real time, detection of minimal residual disease and prognosis for malignant neoplasms. The objective of this study was to present a literature review to clarify the fundamental molecular and clinical aspects involved in this revolutionary diagnostic technique by extracting the data from the sample.
\end{abstract}

Keywords: Liquid Biopsy. Oncology.: literature review

\footnotetext{
${ }^{1}$ City Hall of Custodia, Custodia, Pernambuco, Brazil. Corresponce Author: marlos_duarte@outlook.com;

2 Faculty of Medicine Estacio of Juazeiro do Norte (Estacio-FMJ), Juazeiro do Norte, Ceara, Brazil.

${ }^{3}$ Institute of Mama of Cariri, Juazeiro do Norte, Ceara, Brazil.
} 


\section{Introduction}

Advances in the understanding of molecular biology of cancer have led to the development of the Liquid Biopsy, which is a revolutionary new and reproducible technique, serving for the early detection of malignant neoplasms, as well as for the initiation of personalized and precise treatment, analysis of treatment response in real time, in addition to the prognosis of patients. ${ }^{1-20}$ This tool is currently called the Net Biopsy and uses the detection of biomarkers of cancer cells, such as circulating tumor cells (CTCs), circulating cell DNA (cfDNA), DNA of tumor cells (ctDNA) and exosomes, which can be detected in various fluids, such as blood, plasma and serum, urine, saliva and cerebrospinal fluid (CSF) . ${ }^{2-}$ ${ }^{11}$ This new diagnostic technique can be performed non-invasively or minimally invasive and may offer many advantages over conventional tissue biopsies. ${ }^{1-4,6,7,10-20}$ This new technology can develop a new concept of individualized or personalized therapy, favoring an understanding that the same type of cancer and in the same stage of disease may have different treatments in different patients, because the liquid biopsy may also verify the heterogeneity and some types of tumor mutations and resistance to some types of drugs used in chemotherapy. ${ }^{2,4,5,8,9,17}$

This approach, however, requires the development of reliable biomarkers and biosensors. ${ }^{2,4}$ Conventional biopsies are often invasive, sometimes impractical and many others technically challenging, while liquid biopsies can be performed serially. ${ }^{2,}$ It is important for the physician to analyze the advantages of the liquid biopsy, but also its disadvantages, especially the difficulty of access to the examination and the still frequent cases of false negative situation, which can be caused by inadequate pre-analytical conditions. ${ }^{3}$ Thus, the clinical impact of the net biopsy should be translated through the standardization of the assay, followed by analytical validation. ${ }^{7}$

Therefore, for this analysis, one should ask: what are the main biomarkers and types of fluids used for the liquid biopsy and the main advantages of using this new tool in the clinical, diagnostic, therapeutic and prognostic context compared to conventional biopsy? The purpose of this study is to perform a literature review to elucidate, through the sample data, these fundamental questions that involve this revolutionary diagnostic technique. 


\section{Method}

This is a literature review whose content was studied in the PubMed database, considered the main one in Medical Education, using the following search: 1) use of the descriptors "liquid biopsy", 2) the use of the Boolean operator "And", and finally, 3) the keyword "advances". The research was carried out for the first time on June 14, 2018, after June 15, 2018 and, finally, June 16, 2018, obtaining the same results (129 articles).

After the search, the inclusion criteria were determined by consensus among the authors. The inclusion criteria were: 1) to have a title in agreement with the researched subject, that is, advances in liquid biopsy nowadays; 2) have been published in journals and in English, 3) have been published in the period between January 19, 2018, which was the day of publication of the largest randomized study on Net Biopsy published in the journal Science21, until June 16, 2018, which was the last day of the survey in the PubMed database. With this, the criterion of exclusion was also determined by consensus: to be published as an editorial, comment or letter to the editor.

For the research and selection of the studies, the articles were analyzed by an evaluation of the main author (AMDM) and the other authors reviewed the search based on the mentioned criteria. There was no disagreement between the searches. A sample of 20 articles was determined in the PubMed database $(n=20)$. The 21 st reference was the publication of the mentioned Sciente Magazine itself and the 22nd reference was mentioned in the study of the 2 nd reference, which is within the sample.

The data extracted from the selected articles were directed to address the advances, innovations, particularities, future aspects, advantages of the use of the liquid biopsy in the clinical oncological practice: 1) Biosensors and biomarkers used in the liquid biopsy; 2) Types of fluids used for molecular analysis; 3) Advantages of the liquid biopsy over conventional; 4) Aspects regarding early detection, mutations, prognostic value and treatment follow-up using liquid biopsy; 5) Detection of minimal residual disease (DRM) through the biomolecular method. 


\section{Results and Discussion}

\section{Biosensors}

Biosensors are small devices that use tumor biomarkers as recognition elements or analytes, and are linked to a system of detection, transduction and amplification of the signal generated in the reaction with the target analyte, being in the oncology to detect the characteristics of tumors, as well as obtaining valuable clinical information that can guide treatment. ${ }^{5}$ CTCs, for example, are enumerated from the CellSearch ${ }^{\mathrm{TM}}$ platform, which is the only one authorized by the FDA. ${ }^{2}$ It is crucial that these techniques are studied and developed in order to increase analytical sensitivity. ${ }^{6}$

\section{The biomarkers}

There are several biomarkers used in liquid biopsies, such as circulating tumor cells, free-cell DNA, tumor cell DNA and exosomes. ${ }^{2-5,7,8,10,11,12-20}$ The choice of biomarkers for early detection of cancer is crucial, but it is important to note that they may have high sensitivity and specificity. ${ }^{4}$

\section{Circulating Tumor Cells (CTCs)}

CTCs are cancer cells that have been eliminated to the systemic circulation of primary or even metastatic tumors, which may facilitate the propaedeutic analysis of the presence of metastases, in addition to analyzing tumor heterogeneity. ${ }^{2,5,7,8}$ CTCs are also the main metastatic mechanism of a malignant solid tumor. ${ }^{5}$ They are non-invasive biomarkers that detect cancer early, and provide valuable genomic, phenotypic, and functional information on the prognosis and real-time prediction of response to treatment and mechanisms of drug resistance that are evolving, behaving as the most extensively tested prognostic marker at the time..$^{2,3,7,8}$

CTCs are captured in various ways using immunological recognition based on size or rigidity and other methods of chemical recognition and, once captured, can provide important 120 
information about the dynamic characteristics of the disease without requiring surgical access to the tumor. ${ }^{5,7}$ However, the detection of a CTC is difficult because it is rare in the peripheral circulation, but the sample is positive when there are 1 to 50 CTCs per $7.5 \mathrm{~mL}$ of blood at any time. ${ }^{2,5,7,8}$ Therefore, the count of CTCs has prognostic value before, during and after treatment ${ }^{5}$, and is even superior to other conventional markers such as CEA. In addition, an increase in the CTC count from low to high levels after systemic therapy is associated with a worse prognosis. $^{2}$

\section{Free-cell DNA (cfDNA)}

Circulating cell free DNA (cfDNA) is a biomarker that refers to all DNA fragments deleted by cells (malignant and non-malignant) for the peripheral circulation and can also be used in the liquid biopsy that is generally eliminated from dying cells as part of the cellular tumor, exercise, inflammation or tissue injury. ${ }^{2,7}$

\section{Tumor cell DNA (ctDNA)}

Tumor cells can release DNA fragments into the circulation through apoptosis, necrosis and active secretion, but the former is the most common form of this release. . $^{3,5,7,8}$ Tumor cell DNA (ctDNA) is another promising circulating biomarker which is being developed to complement the study of liquid biopsy; can be very variable and correlate with tumor burden and can provide diagnostic accuracy and predict responses to real-time therapy. ${ }^{2,8,9,11,14,15}$ In addition to having prognostic value, both CTCs and ctDNA are used for molecular analyzes that may guide the individual and personalized selection of therapies, both of which are complementary, but ctDNA is perhaps more appropriate for large-scale serial detection of mutations that may be present, in addition to being more commonly found than CTCs . ${ }^{2,9,11}$

The ctDNA is highly fragmented and represents only a small fraction of the total DNA fragments circulating in the peripheral blood (cfDNA) and may correspond to less than $1 \%{ }^{3,6}$ With this, ctDNA has become a non-invasive cancer biomarker, with applications on the 
detection of metastases and tumor heterogeneity, but this method needs further studies to be able to cover the entire genome and, in addition, have a higher sensitivity. $6,8,9,14,15$

\section{Exosomes}

The exosomes are extracellular vesicles released from tumor cells into the peripheral blood and are also used in this strategy of liquid biopsy, since they may contain microribonucleic acid (miRNA), messenger RNA (mRNA), protein and ctDNA, also having a high value in this new propaedeutics. ${ }^{3,5,7,8,10,17}$ The capture of exosomes on the chip can be useful in the creation of an exosomes database for analysis. ${ }^{5}$

Exosomes can be found in many types of body fluids such as blood, urine, breast milk and saliva, and are actively involved in the development of cancer and the presence of metastasis. Therefore, exosomes, formerly considered as "cell garbage bags" with liquid biopsy, have determined its importance in understanding the molecular mechanisms of cancer biology. ${ }^{10,17}$

\section{Types of fluids for liquid biopsies}

Body fluids can provide, more easily and repeatedly, the pathological characterization of tumors. Such body fluids may be peripheral blood, plasma and serum, urine, saliva, cerebrospinal fluid (CSF), and can be obtained easily and repeatedly from patients and are useful for pathological and molecular characterization by means of " liquid biopsies ". 2-11 Most studies have focused on peripheral blood, serum and plasma, but saliva has been used primarily for lung and oral cancer, whereas CSF in central nervous system cancers and urine for tumors of the treatment urinary tract infection. 5

\section{Blood}

Peripheral blood is the most widely used body fluid for CTC analysis and is a common starting point for deriving plasma or serum for studies involving ctDNA and 
exosomes. Starting with peripheral blood is interesting because it does not require sample preparation between patient collection and analysis. ${ }^{5}$

\section{Plasma and serum}

Both plasma and serum are derived from the peripheral blood without the cell centrifugation process of the blood, therefore not being able to quantify the CTCs because these are already eliminated by the process. Basically, the difference between serum and plasma is that plasma still has anticoagulants present in the blood; therefore, serum requires even more sophisticated equipment to prepare a sample. Thus, in health services, plasma is easier to acquire than serum, although the difference is small, but both are consecratedly used in the net biopsy for a variety of cancers. ${ }^{5}$

\section{Saliva, urine and cerebrospinal fluid (CSF)}

Saliva is composed of water, cells and other organic and inorganic molecular substances that can be interpreted for clinical purposes, being a truly non-invasive method. It is employed especially in the analysis of head and neck cancer and lung cancer. ${ }^{5,8}$

Urine is a non-invasive sample and is most commonly used in the diagnosis and analysis of bladder and prostate cancer, which are urologic tumors. Furthermore, it has been shown that the level of mutant circulating DNA in the urine of patients with non-invasive bladder cancer is directly proportional, linearly, to tumor size and stage. CSF is the fluid that involves the central nervous system (CNS) and requires a relatively invasive procedure for collection, which is lumbar puncture; therefore it should be used only for the investigation of specially selected tumors, which are those of CNS, since the ctDNA captured from the CSF presents better the genomic alterations during brain cancer than the ctDNA removed from the plasma. The obtaining of biomarkers in the CSF in relation to other tumors of other sites can be difficult due to the presence of the blood-brain barrier. ${ }^{5,11}$ 


\section{Net biopsy versus conventional biopsy (tissue)}

Conventional biopsy is currently considered a gold standard test for evidence of cancer. ${ }^{1,2,3}$ However, because it detects gene mutations and non-invasive tumor heterogeneity, the net biopsy may guide the initial treatment, avoiding the use of chemotherapy or immunotherapy that would not have therapeutic response, prescribing the ideal therapy for that type of tumor with those genetic alterations observed in this more current method; in addition to detecting the presence of minimal residual disease after treatment. ${ }^{2,3,5,7,8,10,11}$

Tissue biopsies provide only pathological and molecular information on the parts of the tumor that are not always representative of the global characteristics. ${ }^{3,8,10}$ Tumor heterogeneity may increase even after therapy, which may make it difficult to overcome resistance to treatment. Peripheral blood from cancer patients may contain CTCs and ctDNA, both of which can be used for more accurate tumor characterization. ${ }^{3}$

CTC analyzes may evidence rare subclone genetic changes that are not usually captured in standardized tissue biopsy analyzes. ${ }^{2}$ In clinical practice, it is often difficult to obtain tumor tissue suitable for molecular characterization in tissue biopsies, which creates the need for new interventions surgical procedures to achieve the desired information, while the net biopsy does not have this limitation and still provides more important clinical information, such as the resistance mechanism after the failure of the first-line treatment ("Real-time biopsy").

Despite its advantages over conventional biopsies, net biopsies remain only as an alternative due to the reduced supply of services. ${ }^{3}$

\section{Early detection of cancer, mutation, prognostic value and follow-up of treatment using liquid biopsy}

A French study ${ }^{22}$ used smokers and $3 \%$ of patients with Chronic Obstructive Pulmonary Disease (COPD) presented positivity for CTCs; They were followed up with a low-dose CT scan every year and all patients with CTCs developed lung cancer within one to four years of detection, which resulted in definitive and early surgical resection. 
A prognostic biomarker informs the overall cancer outcome regardless of treatment, while a predictive biomarker provides information on the effect of the therapy performed. Predictive biomarkers, therefore, are based on the molecular characteristics of CTCs and are being used to provide valuable information in order to identify therapeutic targets, mechanisms of resistance and probability of response to specific therapies, allowing the optimization of treatment. ${ }^{2-8,} 10$ Genetic mutations, translocations and rearrangements in chromosomes are some of the innumerable molecular alterations that can occur in tumors and, therefore, the liquid biopsy, when directing the use of drugs indicated for those alterations, can serve to improve the overall prognosis of patients with cancer. ${ }^{2}$ Thus, it is known that serial analyzes of cfDNA during the course of therapy may imply the early detection of the onset of mutations associated with resistance. ${ }^{4}$

\section{Minimum Residual Disease Detection (MRD)}

Minimal residual disease is persistence of tumor at the site of origin, even in small amount, after therapy. Liquid biopsies are also being developed for monitoring therapy and for detecting MRD in early stage cancer patients, but face challenges common to other tests for early detection because of the low concentration of ctDNA in the blood in those early stages of the disease; however, ctDNA detection required studies on tumor-specific mutations and the mutational spectrum, which may change the course of MRD for an evident metastatic disease. ${ }^{4,8,11}$ Currently, most evidence on early diagnosis of cancer and MRD post-treatment, using circulating cancer biomarkers, such as ctDNA, has potentially higher sensitivity compared to other biomarkers such as detectable CTCs. ${ }^{2,4,8,11}$

Although there is evidence to show ctDNA as a promising biomarker for MRD monitoring, early identification of these resistant patients even before the first treatment could improve patient survival, which still needs to be elucidated in large clinical trials. ${ }^{8}$ 


\section{Conclusions and future aspects}

Therefore, it is fundamentally important to evidence the net biopsy as a major advance in the study to assist in the early diagnosis of cancer, as well as the orientation to a personalized therapy, real-time follow-up of the treatment result, detection of minimal residual disease, metastasis and prognosis for the patient.

Liquid biopsy may decrease the role of conventional biopsy in the present day, thus reducing invasive procedures, mainly because it is noninvasive, reproducible, and offers basically all the molecular information necessary to guide the precise treatment of the cancer. The liquid biopsy is performed from biosensors that use the biomarkers as analyte, which can be the circulating tumor cells, tumor cell DNA, exosomes.

These biomarkers can be found in blood, plasma and serum, urine, cerebrospinal fluid, saliva and these can be selected depending on the primary site of the lesion. In addition, it will be imperative for oncology to develop the technique, so that it has more sensitivity, reduces false negatives, and makes the method more present in the services that treat patients with cancer. Liquid biopsy is a great promise, but new studies, of course, are needed to supplement knowledge.

\section{Acknowledgments}

To the Academic League of Oncology of the Faculty of Medicine Estácio of Juazeiro do Norte for the support for the elaboration of this article of literature review.

(*)Conflicts of interest: the authors declare no conflicts of interest.

\section{References}

1. Astaras, C. et al. [Liquid biopsy, a new opportunity for personalized oncology]. Revue Médicale Suisse, v. 14, n. 607, p.1028-1032, 16 maio 2018. 
2. Hwang, William L.; Pleskow, Haley M.; Miyamoto, David T.. Molecular analysis of circulating tumors cells: Biomarkers beyond enumeration. Advanced Drug Delivery Reviews, v. 125, p.122-131, fev. 2018. Elsevier BV. http://dx.doi.org/10.1016/j.addr.2018.01.003.

3. Yoneda, Kazue et al. A liquid biopsy in primary lung cancer. Surgery Today, p.1-14, 11 abr. 2018. Springer Nature. http://dx.doi.org/10.1007/s00595-018-1659-2.

4. Babayan, Anna; Pantel, Klaus. Advances in liquid biopsy approaches for early detection and monitoring of cancer. Genome Medicine, v. 10, n. 1, p.1-3, 20 mar. 2018. Springer Nature. http://dx.doi.org/10.1186/s13073-018-0533-6.

5. Tadimety, Amogha et al. Advances in liquid biopsy on-chip for cancer management: Technologies, biomarkers, and clinical analysis. Critical Reviews In Clinical Laboratory Sciences, v. 55, n. 3, p.140162, fev. 2018. Informa UK Limited. http://dx.doi.org/10.1080/10408363.2018.1425976.

6. Gorgannezhad, Lena et al. Circulating tumor DNA and liquid biopsy: opportunities, challenges, and recent advances in detection technologies. Lab On A Chip, v. 18, n. 8, p.1174-1196, 2018. Royal Society of Chemistry (RSC). http://dx.doi.org/10.1039/c8lc00100f.

7. Morrison, Gareth J.; Goldkorn, Amir. Development and Application of Liquid Biopsies in Metastatic Prostate Cancer. Current Oncology Reports, v. 20, n. 4, p.1-10, 23 mar. 2018. Springer Nature. http://dx.doi.org/10.1007/s11912-018-0683-0.

8. Nonaka, T.; Wong, D.t.w.. Liquid Biopsy in Head and Neck Cancer: Promises and Challenges. Journal Of Dental Research, v. 97, n. 6, p.701-708, 7 mar. 2018. SAGE Publications. http://dx.doi.org/10.1177/0022034518762071.

9. Todenhöfer, Tilman et al. Liquid Biopsy-Analysis of Circulating Tumor DNA (ctDNA) in Bladder Cancer. Bladder Cancer, v. 4, n. 1, p.19-29, 20 jan. 2018. IOS Press. http://dx.doi.org/10.3233/blc170140

10. Liu, Chang; Yang, Yunchen; Wu, Yun. Recent Advances in Exosomal Protein Detection Via Liquid Biopsy Biosensors for Cancer Screening, Diagnosis, and Prognosis. The Aaps Journal, v. 20, n. 2, p.1-13, mar. 2018. American Association of Pharmaceutical Scientists (AAPS). http://dx.doi.org/10.1208/s12248-018-0201-1.

11. Stewart, Caitlin M et al. The value of cell-free DNA for molecular pathology. The Journal Of Pathology, v. 244, n. 5, p.616-627, 12 mar. 2018. Wiley. http://dx.doi.org/10.1002/path.5048.

12. Franchino, Federica; Rudà, Roberta; Soffietti, Riccardo. Mechanisms and Therapy for Cancer Metastasis to the Brain. Frontiers In Oncology, v. 8, p.1-28, 24 maio 2018. Frontiers Media SA. http://dx.doi.org/10.3389/fonc.2018.00161.

13. Yao, Nu et al., E. M. (2018). Structure and function analysis in circulating tumor cells: using nanotechnology to study nuclear size in prostate cancer. American Journal of Clinical and Experimental Urology, 6(2), 43-54.

14. Stewart, Caitlin M.; Tsui, Dana W.y.. Circulating cell-free DNA for non-invasive cancer management. Cancer Genetics, p.1-40, mar. 2018. Elsevier BV. http://dx.doi.org/10.1016/j.cancergen.2018.02.005. 
15. Rohanizadegan, Mersedeh. Analysis of circulating tumor DNA in breast cancer as a diagnostic and prognostic biomarker. Cancer Genetics, p.1-10, fev. 2018. Elsevier BV. http://dx.doi.org/10.1016/j.cancergen.2018.02.002.

16. Torres, Carolina; Grippo, Paul J.. Pancreatic cancer subtypes: a roadmap for precision medicine. Annals Of Medicine, v. 50, n. 4, p.277-287, 22 mar. 2018. Informa UK Limited. http://dx.doi.org/10.1080/07853890.2018.1453168.

17. Halvaei, Sina et al. Exosomes in Cancer Liquid Biopsy: A Focus on Breast Cancer. Molecular Therapy - Nucleic Acids, v. 10, p.131-141, mar. 2018. Elsevier BV. http://dx.doi.org/10.1016/j.omtn.2017.11.014.

18. Wu, Shang-gin et al. Management of acquired resistance to EGFR TKI-targeted therapy in advanced non-small cell lung cancer. Molecular Cancer, v. 17, n. 1, p.1-14, 19 fev. 2018. Springer Nature. http://dx.doi.org/10.1186/s12943-018-0777-1.

19. Gootenberg, Jonathan S. et al. Multiplexed and portable nucleic acid detection platform with Cas13, Cas12a, and Csm6. Science, v. 360, n. 6387, p.439-444, 15 fev. 2018. American Association for the Advancement of Science (AAAS). http://dx.doi.org/10.1126/science.aaq0179.

20. Li, Ting-ting et al. Prognostic and predictive blood biomarkers in gastric cancer and the potential application of circulating tumor cells. World Journal Of Gastroenterology, v. 24, n. 21, p.2236-2246, 7 jun. 2018. Baishideng Publishing Group Inc.. http://dx.doi.org/10.3748/wjg.v24.i21.2236.

21. Kaiser, Jocelyn. 'Liquid biopsy' for cancer promises early detection. Science, v. 359, n. 6373, p.259-259, 18 jan. 2018. American Association for the Advancement of Science (AAAS). http://dx.doi.org/10.1126/science.359.6373.259.

22. Ilie, Marius et al. "Sentinel" Circulating Tumor Cells Allow Early Diagnosis of Lung Cancer in Patients with Chronic Obstructive Pulmonary Disease. Plos One, v. 9, n. 10, p.1-7, 31 out. 2014. Public Library of Science (PLoS). http://dx.doi.org/10.1371/journal.pone.0111597.

\section{How to cite this article (APA format):}

Melo, Antonio Marlos Duarte de; Silva filho, Messias Silvano da; Alves, Bárbara Torquato; Aguilera, Kevellyn Cruz; Alencar, Ana Maria C.; Andrade, Ana Maria L. C. de; Leite, Daniel G.;Quidute, Ricardo Souto. (2018). The Innovations, Advances and Outlooks of Liquid Biopsy in Oncology: a Literature Review. Am In. Mul J, 2 (4), p. 117-128.

Received: 07/04/2018.

Accepted: 07/06/2018. 